\title{
Mapas conceituais e o jogo: estratégias pedagógicas de ensino e aprendizagem de Bioquímica
}

Ana Maria Ponzio de Azevedo Maria Suzana Marc Amoretti Maria Isabel Timm Milton AntonioZaro

\section{Conceptual maps and a game: pedagogic strategies for biochemistry teaching and learning}


Resumo: O artigo relata e analisa a utilizaÁ,o de mapas conceituais aplicados $\ddagger$ avaliaÁ,o de conceitos aprendidos com o uso do jogo Diagrama MetabÛlico Din,mico (DMD) na disciplina de Bioquìmica da FundaÁ,„o Faculdade Federal de Ciĺncias MĖdicas de Porto Alegre. Explora a validade do uso do mapa conceitual como ferramenta pedagÛ́gica, pelo estudante, na modelagem dos conceitos e autoavaliaÁ,,O; e pelo professor, no acompanhamento e avaliaÁ,,o do processo de aquisiÁ,,o de conhecimento pelo aluno. Cada participante, individualmente, construiu um mapa conceitual inicial, sobre um determinado metabÛlito indicado pelo professor. Em seguida, participou de uma atividade em grupo, montando, de forma colaborativa e cooperativa, um jogo sobre este metabÛlito e apÛs construiu, individualmente, novo mapa conceitual. Uma an-lise qualitativa comparativa foi feita entre os mapas conceituais inicial e final de cada participante. O inicial foi usado como indicador do conhecimento prËvio do participante sobre o assunto trabalhado e o final para verificar a expans,,o dos conceitos processados pelo aluno apÛs o jogo. Foram considerados os seguintes pontos: predomin,ncia do car-ter associativo ou classificatÛrio; conceitos e relaÁıes corretas; n' mero de relaÁıes. Os mapas iniciais indicaram que todos os alunos possulam algum esquema mental prËvio sobre o conceito proposto. Todos os mapas conceituais finais indicaram expans, o de conceitos em relaÁ,,o aos mapas iniciais, o que pode ser percebido atĖ mesmo pela observaÁ „o do tamanho dos grafos. A observaÁ,,o puramente visual comparativa entre os mapas mostra que houve uma agregaÁ,,o de novos elementos. Evidenciou-se a predomin,ncia do car·ter associativo em relaÁ „o ao car·ter classificatÛrio. Os resultados apresentados sugerem a validade do uso do mapa conceitual como estratĖgia de acompanhamento do processo cognitivo, pelo professor e pelo prûprio aluno.

Palavras-chave: mapa conceitual, estratĖgia cognitiva, ensino de bioquìmica, construÁ,,o do conhecimento, trabalho colaborativo.

\begin{abstract}
This paper reports on the use of concept maps applied to the evaluation of concepts learned through the use of the game Dynamic Metabolic Diagram (DMD - described in Biochemical Education, vol. 20, n 2, p. 97-98, 1992) in the discipline of Biochemistry of FFFCMPA. An investigation was carried out into the validity of using concept maps as a pedagogical tool both by the student, in the modeling of concepts, and by the teacher, in the assessment of the studentís learning. Each participant constructed an initial concept map about a given metabolite assigned by the teacher. Subsequently, he or she participated in a group activity, playing an educational game about this metabolite, and later the student constructed a new concept map. A comparative qualitative analysis was carried out between the initial and final concept maps of each participant. The initial concept was used as an indicator of the participantís previous knowledge of the topic, and the final concept was used to check the expansion of the concepts by the student after playing the game. The following criteria were used: predominance of associative or classificatory character; correct concepts and relationships; number of relationships. A purely visual comparison between the maps indicated that new elements have been added. The associative character has been shown to predominate when compared to the classificatory one. The results obtained suggest the validity of using concept maps as a strategy to monitor the cognitive process, both by the teacher and by the student.
\end{abstract}

Keywords: concept map, cognitive strategy, biochemistry teaching, knowledge construction, collaborative work.

AZEVEDO, Ana Maria Ponzio de et al. Mapas conceituais e o jogo: estratĖgias pedagÛ́gicas de ensino e aprendizagem de Bioqulmica. Informática na Educação: teoria \& prática, Porto Alegre,v.7, n. 1, p. 5971, jan./jun. 2004. 


\section{Apresentação}

ODMDÈbaseado numjogo deseq, Íncia IÛgica, que vem sendo usado desde 1991 na disciplina de Bioquìmica da FFFCMPA. O jogo, na sua primeiravers,,o, foi documentado na revista Biochemical Education (SIQUEIRA, 1992). Nestaexperiĺncia, procurouse associar ao uso do DMD a aplicaÁ,,o de mapas conceituais, comoestratĖgiadeensino e aprendizagem, tendo como objetivos gerais explorar as possibilidades de uso dessas ferramentas derepresentaÁ,,odeconceitosesuas relaÁıes para avaliaÁ,,o do conhecimento do aluno. Para isso, foielaborada pesquisa experimental com dois grupos de estudantesquej. tinham cursado ou ainda estavam cursando a disciplina deBioqul̀mica. Foramfeitas an-lises de mapas conceituais elaborados pelo aluno antes e depois do jogo. $\mathrm{N}_{\text {, }} \mathrm{O}$ ser,,O considerados neste artigo elementos relacionados $\ddagger$ tecnologia de produÁ,,o e operaÁ,,o do jogo e de realizaÁ,o dosmapasconceituais.

\section{Introdução}

Promover a aprendizagem do aluno È objetivo principaldo professor. Napr-ticapedagÛgica, Èimportanteoprofessorconhecercomo ocorreaaprendizagemerefletirsobreseupapel como mediador do processo de aprendizagem doaluno. AgrandequantidadedeinformaÁıes, emtodasas reas, tornaoaprendizadodenovos conte dosilimitado.

Dessa forma, a tarefa do professor, inclusive no ensino superior, excede a simples transmiss,,o do conhecimento e adquire um car-ter muito mais formativo que informativo. Paraisso, necessita estabelecer estratĖgias de ensino voltadas a estimular a aÁ,o do alunoe seu envolvimento comos conte dos propostos.
Oconceito de estratégiautilizadoneste trabalhoÈode Fayard (2000): ìcobreaconcepÁ,,o, a geraÁ,,o ea implantaÁ,,o de vias e meios queconcretizamumobjetivoperseguidoî(p.32). InformaÁ,,o ecomunicaÁ,,o representam, portanto, os meios de realizaÁ,,o daestratĖgia definidaparaatingiroobjetivo definido peloestrategista, que, nesse caso, ser· identificadocom oprofessor, cujoobjetivofinalĖapoiaraatividadedeaprendizagem doaluno.

No ensino, o professor usa de v-rias estratÈgias para representare disponibilizaro conte do de sua disciplina, sem o que a informaÁ,,o pode ser percebida pelo aluno como uma simples teia de conte dos complexos e incompreensìveis, o que muitas vezesocorre no ensino de Bioquilmica, que trata das subst,ncias e suas transformaÁıes em nìvel celular. Nesse caso, È preciso disponibilizar a informaÁ,o necess·ria para o entendimento do conte do, procurando modelar de forma mais eficiente o conhecimento que o aluno dever. construir, incluindoarede deconceitosenvolvidos nesse conte do.

Para apoiar este 'Itimo item ñ a aprendizagem da rede de conceitos envolvidos no conte do de Bioquìmicañ foram usados mapas conceituais, ferramentas sugeridas pelas ciĺncias cognitivas. O objetivo deste trabalho È descreveruma experiĺncia pr-tica de uso de mapas conceituais no ensino de Bioquìmica, visando acompanharo processo de aprendizagem dos alunos, monitorado atravĖs de sua capacidade de expressar os conceitos da disciplina, ao qual tiveram acesso atravÈs de um estudo em grupo com jogo de seq,Íncialûgica.

\subsection{Mapas conceituais e o ensino de Bioquímica}


Osmapasconceituais(MOREIRA, 1987) s, ,o representaÁıes gr·ficas de um conjunto deconceitos dequalquerdomìnio, disposto de forma a evidenciar suas relaÁıes. S, ,o diagramas que facilitam a modelizaÁ,,o do conhecimento, tendo sido usados em v rias ·reas das Ciĺncias Cognitivas. Podem serusados como um instrumento que se aplica ao ensino e aprendizagemescolar, desdeas atividades de an-lise de currìculo, atĖ a produÁ,,o de sistemas virtuais, em avaliaÁ,,o e pesquisas em monitoramento dos processos educacionais. Estruturados a partir das teorias relacionadas ‡sredessem,nticas(AMORETTI,2000), podem representaruma estruturahier-rquica, quevai desde os conceitos mais abrangentes atÈ os menos inclusivos, como foi proposto no presente trabalho, ou de forma puramente relacional, sem qualquer hierarquia entre os conceitos. Podem serutilizados paraauxiliara ordenaÁ,,o e o seq, enciamento hierarquizado dos conte dos de ensino, de forma a permitir ao aluno organizar seus conhecimentos. Os mapas podem serdefinidos pela predomin, ncia do car.ter puramente associativo dos conceitos apresentados (atributos); ou do car-ter classificatÛrio (categorias).

No caso dos mapas associativos, aplicados ao tema da Bioquìmica, considerou-se que apenas estabelecem relaÁıes entre os conceitostratados, as propriedades dassubst,ncias e suas reaÁıes, de forma n,o hier·rquica ou classificatÛria. Durante a experiĺncia observada, foramconsiderados associativosos mapasqueapenas descreveramconhecimento sobreoassunto, associando conceitos sem estabelecer categorias, nìveis, ordens ou etapas de processamento. J. os mapas de natureza classificatória, nessecaso, foramconsideradosaqueles nos quaiso aluno estruturouconhecimentoatravĖsdadefiniÁ,,odecategorias, identificando ordem, etapas e hierarquia no processamentodasreaÁıes.

Ouso de mapas conceituais possibilita apoiar o desenvolvimento de um processo cognitivo de aprendizagem em que o aluno organiza o seu conhecimento de forma autÙnoma. Ele prÛprio orienta a aquisiÁ,,o de novas informaÁıes que estar, ,o diretamente relacionadas com o conhecimento que possui no momentodaaprendizagem. NaelaboraÁ,,ode um mapa conceitual, o aluno aprende a agrupar os conceitos segundo seus traÁos perceptivos e segundo as categorias que tím um significado em sua experiĺncia anterior. EssarepresentaÁ,,ofacilitaa apreens,„o doconhecimento porque a memÛria humana reconheceeretĖmmais rapidamenteas propriedadesmais importantes doconceito, facilitandoo processo mental dacompreens,,o.

O fato de simular aspectos tìpicos da cogniÁ,,o humana, atravĖs da associaÁ,,o de novas propriedades aos conceitos b-sicos, constituiumarepresentaÁ,,o aberta do conhecimento(AMORETTI,2000). ComorepresentaÁ,o aberta do conhecimento, n,o existe mapa certo ou erradoe, porisso, a avaliaÁ,,o dotrabaIho do aluno $n$, o pode ser feita pelos par,metros tradicionais de mensuraÁ,,o por perguntase respostas. Naverdade, essesinstrumentos $\mathrm{n}, \mathrm{o}$ podem ser usados como ferramentadeavaliaÁ,o, mas de monitoramento de percursocognitivodoaluno. Sobretudo, enquanto ferramenta de apoio, dever-sero produto da capacidade doaluno de expressarasconexies entreosconceitose responderporelas, deformalÛgica.

Entre as funÁıes do mapa conceitual podem-se destacar:a de descreverosobjetos daexperiĺncia; afunÁ,,oeconÙmica, vinculada ao car·terclassificador; a funÁ,,o de organizar os dados da experiĺncia de modo que se estabeleÁamentreelesconexıesIÛgicas; eaindaa 
funÁ,,o fundamental de previs,„O, na medida em que o conceito $\dot{E}$ um meio antecipador (AMORETTI,2000).

Segundo Moreira (1987), o mapa conceitual È um recurso que visa a facilitar a aprendizagem deconceitos. Dispensaequipamentossofisticadosou instalaÁıes especiais, possibilitando seu uso atÈ mesmo nas mais modestas condiÁıes de trabalho. Os mapas conceituais podem ser usados, como recurso did·tico, para organizar relaÁıes entre conceitos que integram o conte" do de uma 'nica aula, de uma unidade de estudo ou de um curso inteiro.

Um exemplo de uso de mapas conceituais em Bioquìmicapode serencontrado no livro de Campbell (2000). A autora apresenta, no inl̀cio de cada capl̀tulo, um mapa conceitualsobreoassunto que ocapl̀tuloaborda, proporcionando ao leitor uma vis,,o geral dos pontos e suas relaÁıes, conforme ser,„O tratadas nolivro. Este exemplod· umaidÈia da natureza visual daferramentae desuacapacidade de representar um conjunto, as partes e suaintegraÁ,,ono todo. ${ }^{1}$

\section{Relato da experiência}

Utilizando uma estratÈgia de pesquisa experimental, o estudo foi realizado com dois grupos de volunt -rios que mostraram interesse em aprofundar seu conhecimento em Bioqulmica. Todos os participantes j tinham tido aula teÛrica, expositiva, sobre o assunto a ser trabalhado, masnenhumdelesconheciamapa conceitual.

O primeiro grupo foi composto por sete alunos do curso de Biologia/UFRGS, que n,,o conheciamoDMD.Oassuntofoiescolhidopelo professor. O segundo grupo foi composto por quatroalunos docursodeMedicina-FFFCMPA e monitores da Disciplina de Bioquìmica, que j· tinham utilizado o mÈtodo do DMD, mas $n, 0$ o referente ao assunto escolhido pelo professor e diferente do assunto trabalhado com o primeiro grupo. O primeiro grupo trabalhou o conceito deUrÈiae osegundo de Fenilalanina.

Comoosparticipantesnuncatinham trabalhado com mapas conceituais, o professor fez uma explicaÁ,,o sobre o conceito e uso do mapacomorepresentaÁ,,odoconhecimentoe entregou paracadaumomapaconceitualapresentadonaFigura 1.

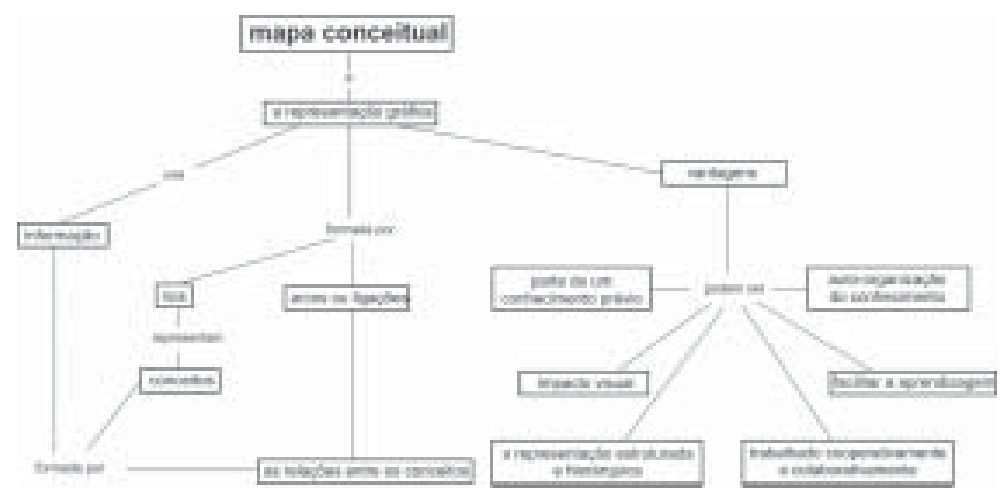

Figura 1: Mapa conceitual explicativo, entregue para os participantes

\footnotetext{
${ }^{1} \mathrm{~N}_{\text {„o }}$ foram localizadas outras referíncias a respeito de uso de mapas conceituais no ensino de Bioquìmica.
} 
As atividades ocorreram durante duas sessies. Na primeira delas, cada participante construiu seu mapa conceitual inicial, individualmente, nomomento escolhido porele, sema presenÁa do professor e dos demais participantes. $^{2}$

O grupo se reuniu em dia e hora estabelecidos e o professor coordenou a aplicaÁ,,o do jogo DMD sobre o conceito contido no mapa conceitual inicial, colocando-se como mediador do trabalho dos alunos com o conceito, para realizar as atividades dojogo. ApÛs o estudo em grupo, os participantes foram deixados livres para repetir o jogo, discutir o conte do entre eles, sem a presenÁa do professor e, na seq, Íncia, elaborar, individualmente, o mapa conceitual final.

Para a construÁ,,o do mapa conceitual foram utilizados apenas papel, I-pis e canetas. O professor $\mathrm{n}, \mathrm{O}$ analisou o mapa inicial antes de coordenar a aplicaÁ,,o do DMD. Apenas assinalou como mapa Iniciale, ao tÈrmino da atividade, devolveu-o para cada participante fazer o mapa conceitual final, com base no mapa anterior.

Umaan-lise qualitativa comparativafoi feita entre os mapas conceituais inicial e final de cada participante. Foram considerados, nesta an lise, os seguintes pontos: predomin,ncia do carter associativo ou do car·ter classificatÛrio; conceitos e relaÁıes corretas; coeríncia; $n$ ' mero de relaÁıes; criatividade e IÛgica.

\section{Resultados e discussão}

\subsection{Observações gerais}

\subsubsection{InformaÁ,o visual}

Osmapasconceituaisdos 11 participantes foram reunidos e est,,o disponìveis em $<$ http:bioquimica.fffcmpa.tche.br/mapas.htm>. AlgumasobservaÁıesgeraispuderamserrealizadas a partir da an-lise do conjunto. Os mapas iniciais indicam que todos os alunos possulamalgumconhecimentoprËviosobreoconceito proposto. Todosos mapas conceituais finais mostraram uma expans,,o dos conceitos em relaÁ,o aos mapas conceituais iniciais, o que pode ser percebido atÈ mesmo pela observaÁ,,odotamanhodosgrafos. AobservaÁ,,o puramente visualcomparativaentreosmapas iniciais e finais de cada participante mostraque houve umaagregaÁ,,o de novoselementos, reforÁando a oportunidade do uso de mapas conceituais na aprendizagem. Os alunos com menorconhecimentoprÈvio (menosrepresentaÁıes no mapa inicial) apresentaram maior expans,,o do mapa conceitual final.

A sinalizaÁ,,o pelo professor, em amarelo, de um conceito inadequado no mapa, mostra alguma falha na aprendizagem do aluno em relaÁ,,o a um determinado conceito. Este È um excelente recurso para avaliar o conhecimento do aluno e facilita o trabalho do professor em relaÁ,,o ao conceito. PÙdese observar que os alunos do curso de Medicina apresentaram maior conhecimento do assunto (apenas um participante apresentou um conceito inadequado).

\subsubsection{Instrumento de auto-avaliaÁ,,O}

AIÈm de representar o conhecimento prÈvio e o adquirido pelo aluno apÛs o jogo, justificando a aplicabilidade da ferramenta como estratĖgia de acompanhamento do processo cognitivo, pelo professor, os mapas conceituaistambĖmsetransformaramemins-

${ }^{2}$ ApÛs o jogo, outro mapa deveria ser construlddo pelo aluno, para comparaÁ,o entre os dois. 
trumento importante para a auto-avaliaÁ,,o do prÛprioaluno. AvaliaÁıesoraisfeitas pelosalunos foram determinantes para essa observaÁ,,o. Algunsalunos relataramquefoidifìcilfazer oprimeiro mapae, apÛso jogo, foi f · cil fazer o segundo ${ }^{3}$. Tal postura remete $\ddagger$ consciĺncia do aluno sobre o seu processo de aprendizado, cujo indicador È o prûprio mapa.

\subsubsection{Inter-relaÁ„,oentreosconceitos}

Os mapas tambÈm apresentam outra caracterl̀stica desej-vel ao processo de ensino-aprendizagem:ainter-relaÁ,,oentreconceitos, de tal formaquen,o se caracterizemcomo fragmentos, massimcomo partes de um todo, cujas relaÁıes s, ,o fundamentais para a compreens, ,o do conte do em seu sentido mais complexo, que inclui generalizaÁıes, abstraÁres e disposiÁ,,o de conte dos por ordem de relev,ncia e sentido. Em todos os mapas conceituais evidencia-se a predomin,ncia do car·ter associativo em relaÁ,,o ao car·ter classificatÛrio.Porexemplo, nomapaconceitual da uréia foram associados os conceitos de local de formação, função, composição, o que d. uma perfeita idÈia da inserÁ,,o do conceito em umconjuntocontextualizado.

\subsection{Relatos de observações particulares}

Embora os assuntos tenham sido diferentesentreosdois grupos, pÙde-se perceber que os conceitos foram agrupados segundo categoriasrelacionadas $\ddagger$ experiĺnciaprËviado autordo mapa.J.nas representaÁıesiniciais, percebeu-se que os alunos do curso de Biolo- gia tratam do assunto com enfoque diferente dosdocursode Medicina. Porexemplo, nogrupo de alunos de Biologia, foi apresentada em trís mapas ${ }^{4}$ umarelaÁ,,odoconceitourÈiacomo fertilizante, benĖficaparaasplantas. J.nosseus mapasfinais, as relaÁıes doconceitourÈia, que $\mathrm{n}$,,oforam trabalhados nojogo(DMD), sugeresequedevem terconsiderado irrelevantesestesconceitos naquelemomento, porque autilidade do metabÛlito n,o era o foco do estudo,e sim a sua sìntese.

AlÈm disso, tambÈm houve uma evidÍncia de funÁ,o antecipadora do mapa, pela an.lise da participante 3. No primeiro, ela usou um conceito errado, que foi desconsiderado na vers,,o final. Supre-se que a aluna atualizou o conceito.

A observaÁ,o dos mapas conceituais elaborados pelo participante 2 mostra o estabelecimento de novas relaÁıes a partir dos conceitos j- existentes. Por exemplo, no primeiro escreveu apenas o local onde a UrÈia È formada e no segundo indicou o caminho percorrido e as transformaÁıes que sofrem os compostos para sintetizar a UrÈia. Por tratar-se de um bom exemplo da facilidade de observaÁ,,o visual da expans,"o de conceitos, e, pela indicaÁ,,o de complexidade da relaÁ,,o percebida entre eles, a Figura 2 apresenta os mapas inicial e final desse participante.

Conceitos bioquimicamenteerradosforam suprimidos no mapa conceitual final do participante 4, sem que o professor tenha feito acorreÁ,,oou discutido este conceito duranteo estudo com o DMD. Os mapas conceituais fi-

\footnotetext{
${ }^{3}$ Embora o prÛprio DMD n,o tenha sido objeto de avaliaÁ,o nesse momento, do ponto de vista da eficiĺncia do jogo como estratĖgia educacional, a expans, o do mapa conceitual inicial de todos os participantes permite sugerir que o DMD facilita a aprendizagem, uma vez que possibilita ao aluno fazer novas relaÁıes e ampliar o seu conhecimento.

${ }^{4}$ Disponibizados como mapas 3, 4 e 5 no endereÁo j· indicado no item 3.1.1.
} 


\section{teoria \& prática}

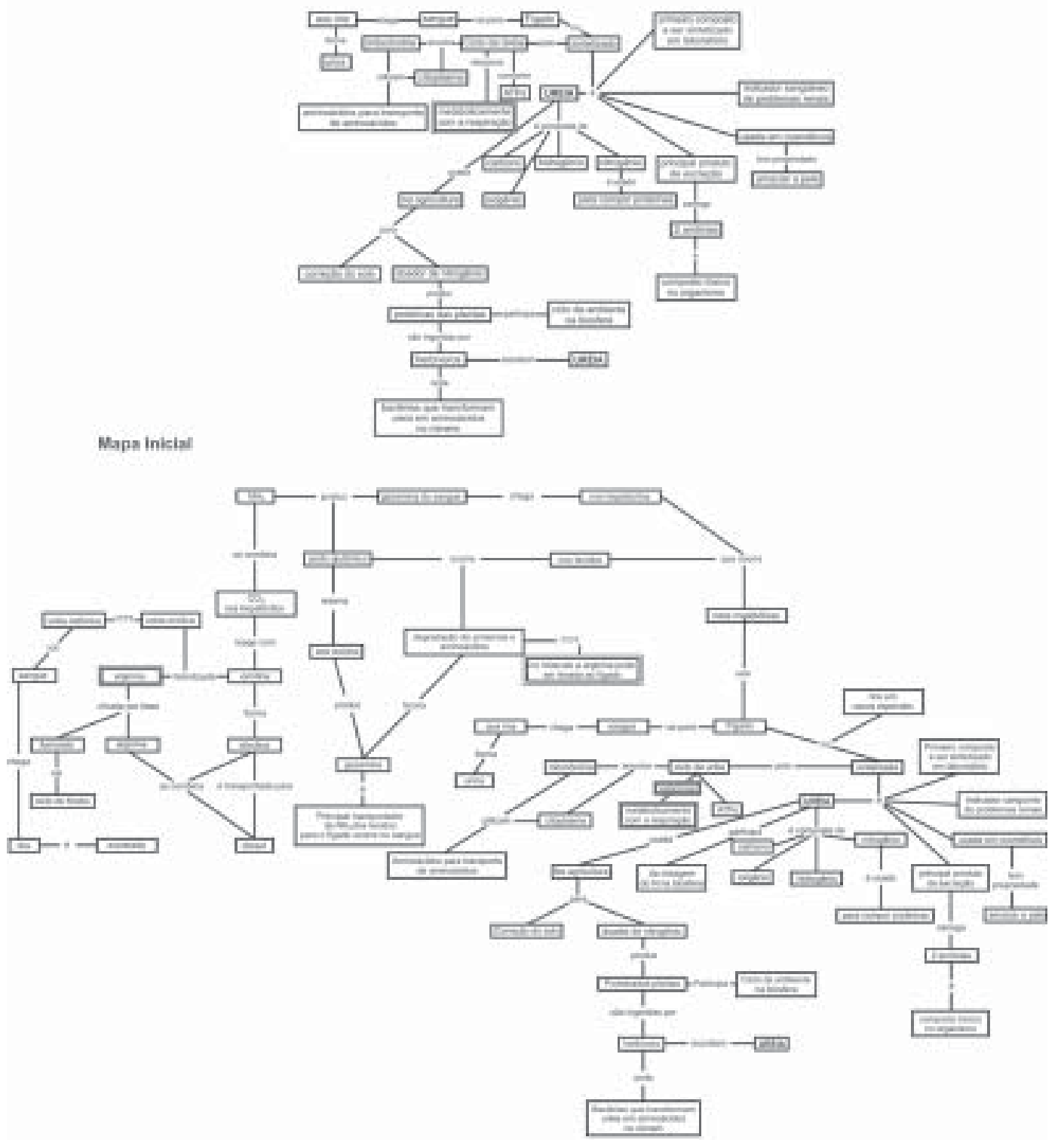

Figura 2: Mapas conceituais do participante 2. O inicial $\ddagger$ esquerda e o final $\ddagger$ direita

Mapa Final

nais de todos os participantes mostramoestabelecimentodenovas relaÁıesapartirdosconceitos j existentes, mesmo naqueles em que omapaconceitualinicialfoiconsideravelmente amplo, identifica-se novas relaÁıes como È o casodoparticipante 11, apresentadonaFigura3.

Emalgunscasosoparticipanteapresentou dificuldades em colocar os links entre os 


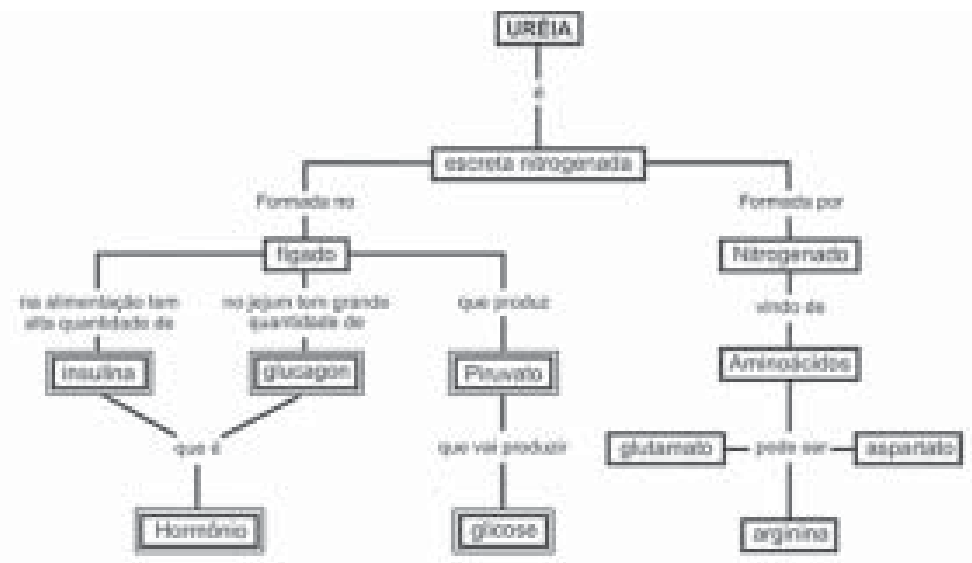

Mapa Inicial
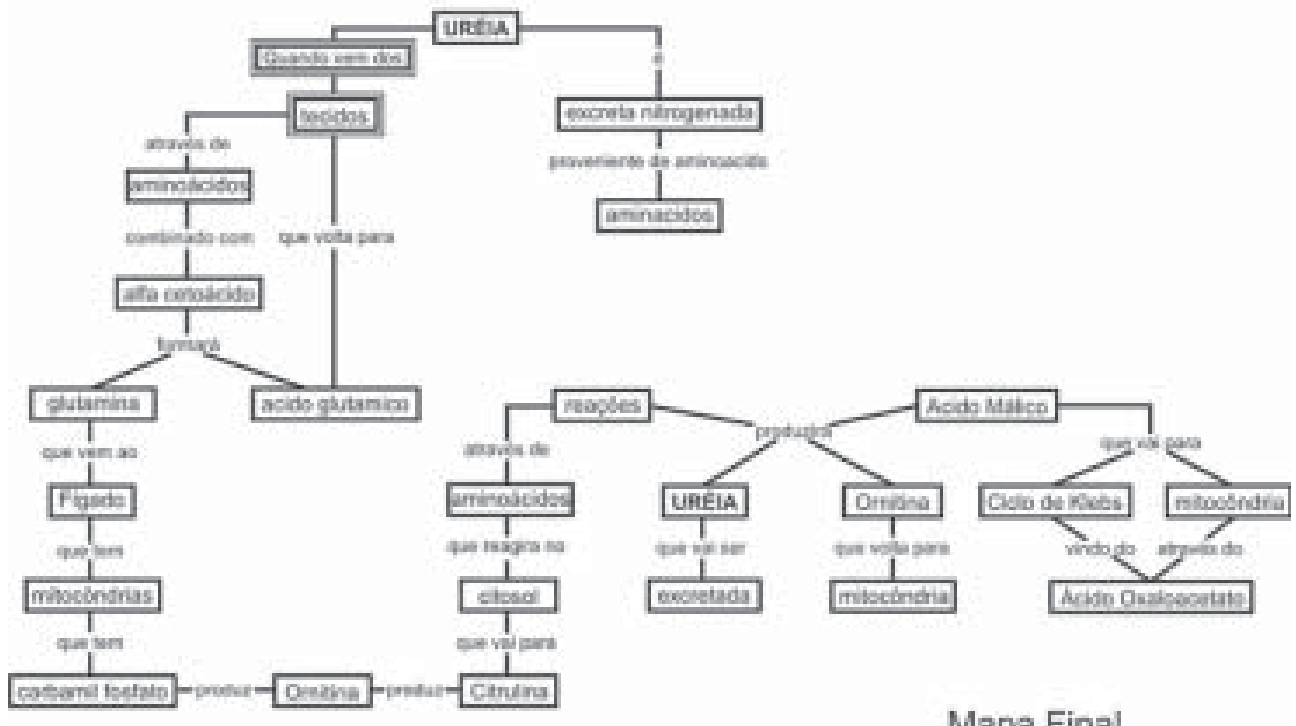

Mapa Final

Figura 3: Mapas conceituais do participante 4.

conceitos, ou os omitiu. Por exemplo, o mapa inicial do participante 8, em quen,oforamcolocados os links. Esta dificuldade $\mathrm{n}, 0$ apareceu nos mapas conceituais finais, o que pode ser explicado pela falta de experíncia dos participantesemelaborarmapasconceituais. 


\section{teoria \& prática}

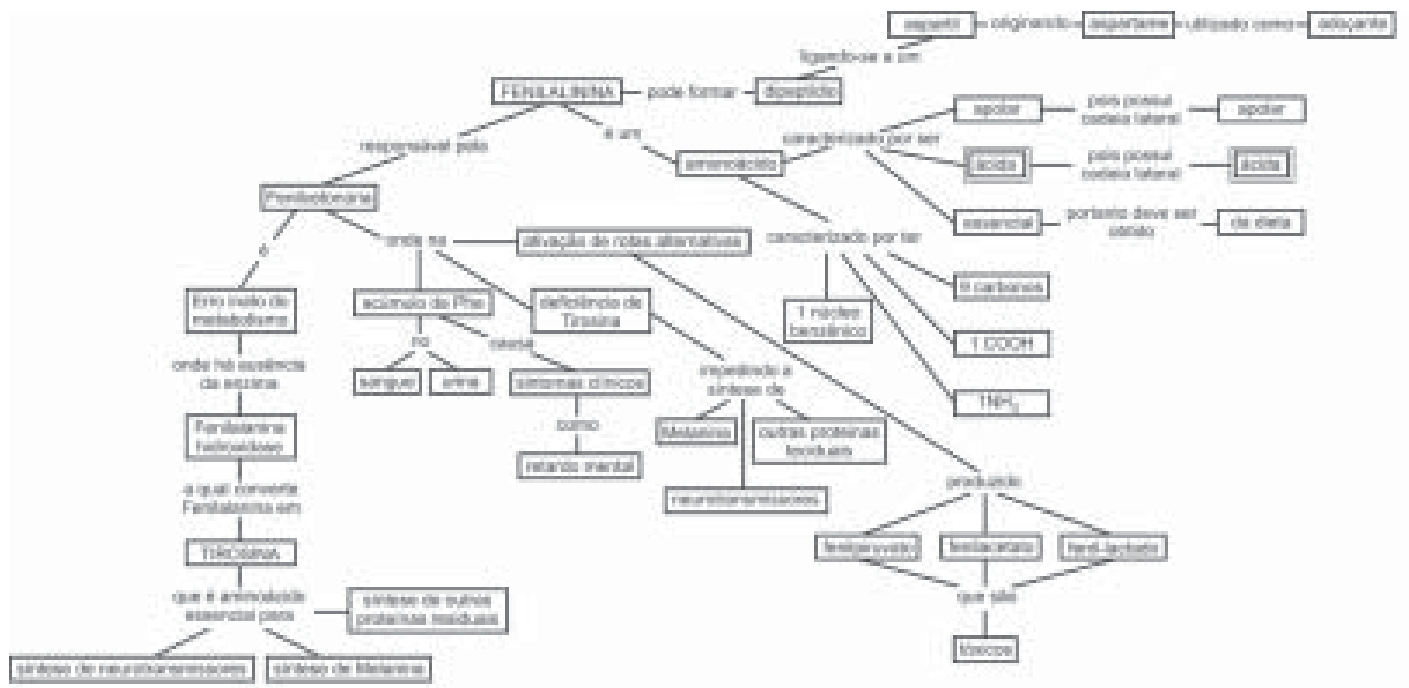

Mapa Inicial

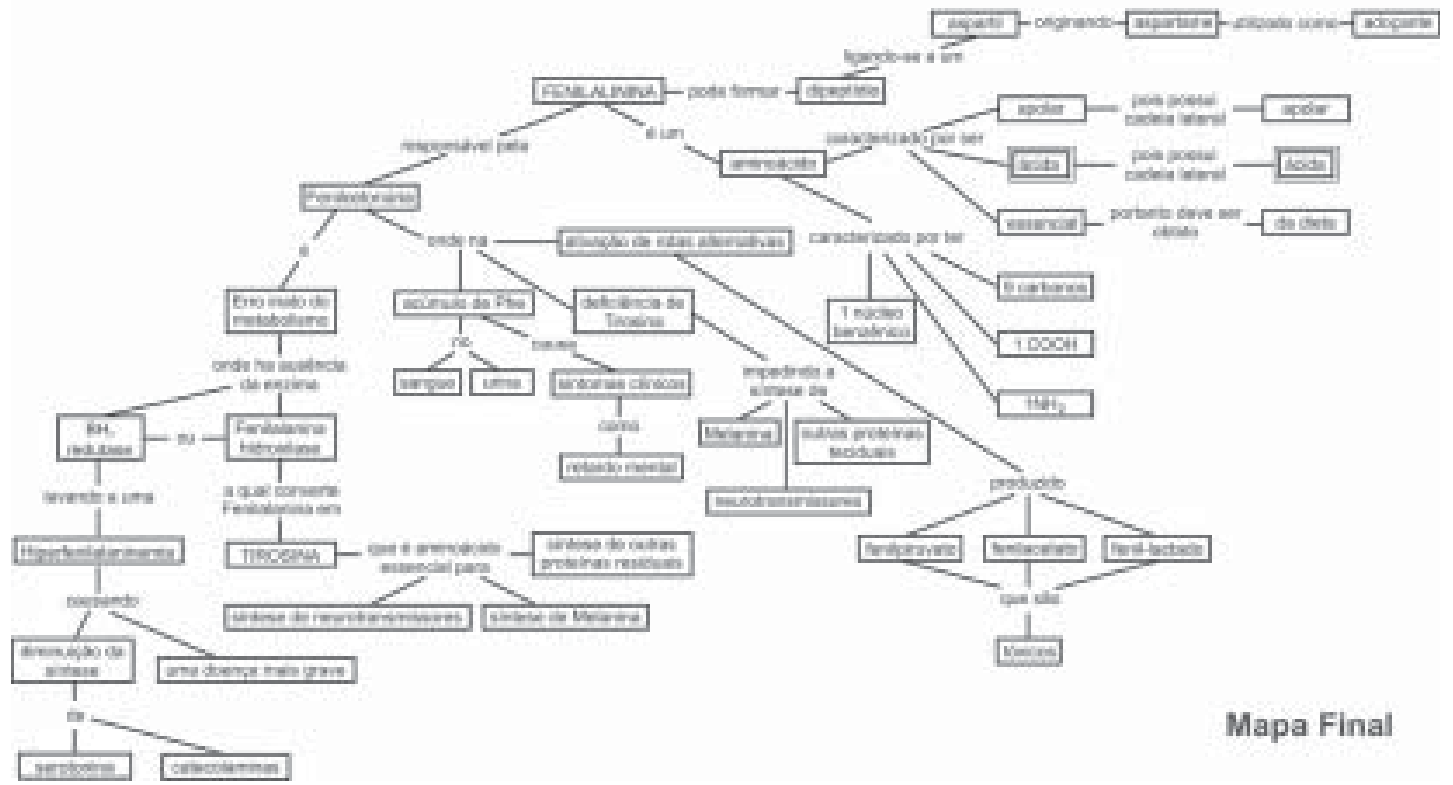

Figura 4: Mapas conceituais do participante 11. 

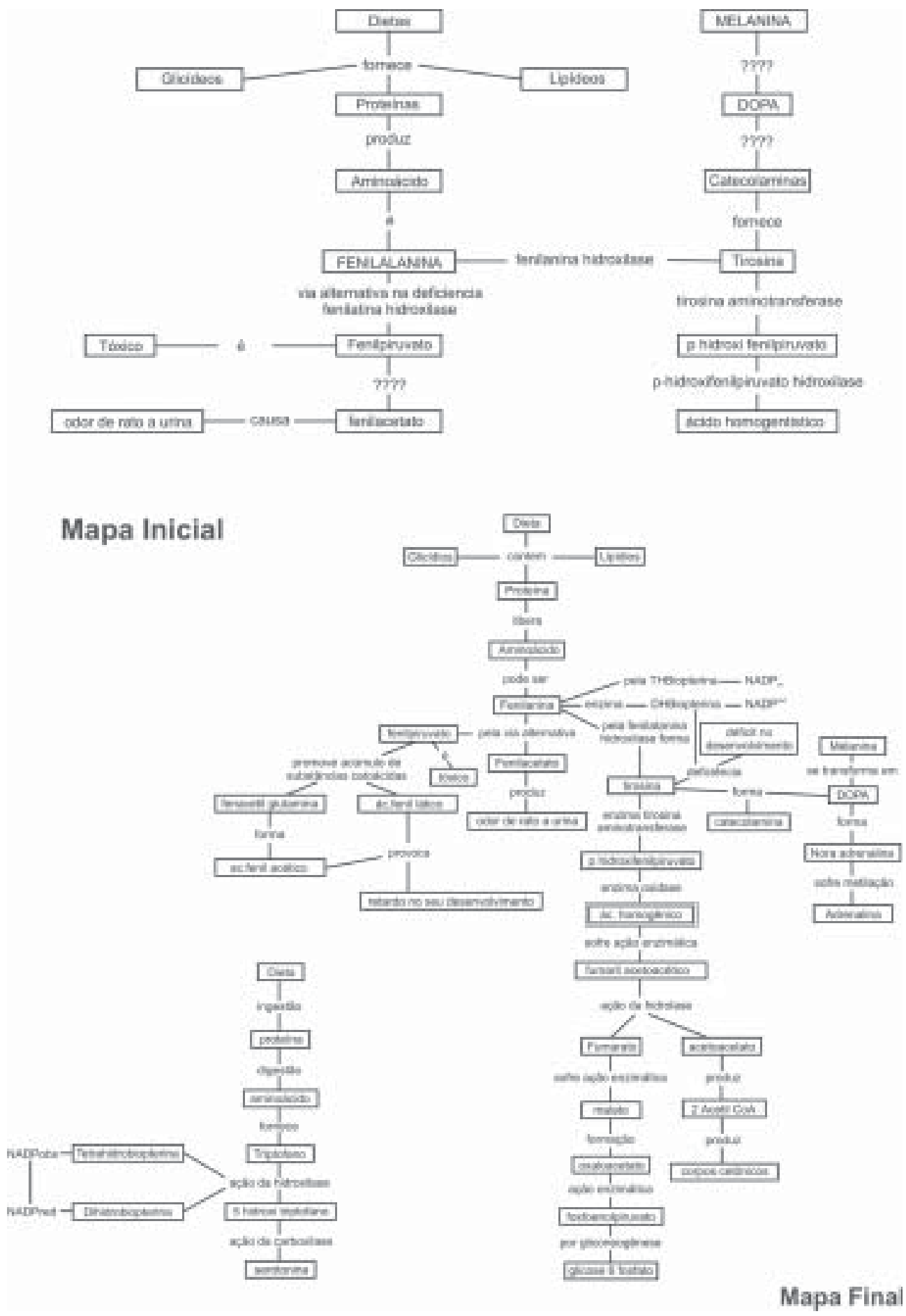

Figura 5: Mapas conceituais do participante 8. 


\section{Conclusão}

AcomparaÁ,,oentre os mapas iniciaise finais de cadaparticipante permiteconcluirque houve expans,,o dos conceitos e de relaÁıes entre eles, evidenciadas pelos mapas conceituais.

Pelos resultados relatados È ineg $\cdot$ vel queautilizaÁ,o de mapas conceituais auxiliaa observaÁ,,odaaprendizagemdenovosconceitos. Sugere-seque possam terocorridoalteraÁıes naestruturacognitivadosalunosquetestaram seu conhecimento prÈvio, realizaram o jogocomoatividadepedagÛgicaeapÛs representaram novamente seu conhecimento em novos mapas conceituais.

Os mapas mostraram que os alunos modificaram a compreens,,o sobre os conceitos que constitulamo assunto ${ }^{5}$, alÈm de terem formandonovas conexies entreeles. Aaprendizagem teria ocorrido a partir da conscientizaÁ,,odoalunosobreseuprÛpriopro- cesso de construÁ,o da teia de conceitos representadaatravĖsdosmapas conceituaisiniciais e finais.

AlÈm disso, a an.lise dos mapas conceituais dos alunos permite ao professor rapidamente identificar $\mathrm{n}, \mathrm{o}$ apenas os pontos em que um determinado aluno n,,o apresentou um bom entendimento, mas tambÈm ter uma vis,,o do conjunto da turma e realizar um planejamento de partes do curso, ou mesmo do prÛprio jogo, que podem ser reformuladas para facilitaracompreens,,o.

O trabalho concluiu n,o apenas pela utilizaÁ,,o bem sucedida desse instrumento de representaÁ,,o e monitoraÁ,,o do processo cognitivo dos alunosño mapa conceitual ñ, mas tambÈm, indiretamente, pela an.lise dos resultados apresentados nos mapas, pela eficiĺncia dojogoDMD como estratĖgia pedagûgica eficiente para o ensino de Bioquilmica, devendo ser realizadas novas experiĺncias para validar ambas as conclusıes.

\footnotetext{
${ }^{5}$ Os autores ressaltam que o instrumento foi usado imediatamente apÛs a realizaÁ,o do jogo, n„o tendo sido
} avaliada a consolidaÁ,o do conhecimento adquirido em termos de memÛria de longo prazo. 
Agradecimentos

AgradecemosaosalunosdocursodeMedicinadaFFFCMPAeaosalunosdocursodeBiologiadaUFRGS, porteremaceitoparticipar destetrabalho; aoSrRubens Renato Abreupelotratamentodeimagens, especialmente $¥$ TÈcnicade LaboratÛrioeacadímica de BiologiaRosangelaBjerk, peloseuauxìlionaexecuÁ,,odestetrabalho;ao ProfessorAntÙnio Jo,oS·de Siqueira, porsuaconstante orientaÁ,,oełsecret·riaAnaLuizaOliveira.

Referíncias

AMORETTI, Suzana Marc. ProtÛtipos e estereÛtipos: aprendizagem de conceitos Mapas Conceituais: experiÍncia em EducaÁ„,o a Dist,ncia. Revista Informática na Educação Teoria \& Prática, v.4 n.2: PGIE/UFRGS, dez 2001.

AMORETTI, M.S.M.; TAROUCO, L.M.R. Mapas Conceituais: Modelagem Colaborativa do Conhecimento. Porto Alegre, Informática na Educação: Teoria e Prática, v. 3 n.1, p.67-71, set 2000.

CAMPBELL, M.K. Bioquímica. 3a. EdiÁ,o. Porto Alegre: Artes MĖdicas Sul, 2000.

FAYARD, P. O Jogo da Interação. InformaÁ,o e ComunicaÁ,o em EstratÈgia. Caxias do Sul: Universidade de Caxias do Sul, 2000.

MOREIRA, Marco A. Mapas Conceituais - Instrumentos Didáticos, de Avaliação e Análise de Currículo. S,o Paulo: Moraes, 1987.

SIQUEIRA, A.J.S.; REMIVO, J.O.R., AZEVEDO, A.M.P. Dynamic Metabolic Diagrams in Biochemistry. Leeds, Biochemical Education, v.20 n 2, p. 97- 98, 1992.

Recebido em 01/09/2003

AceitoparapublicaÁ,,oem24/07/2004

\section{Ana Maria Ponzio de Azevedo}

Professora da FundaÁ,o Faculdade Federal de Ciĺncias MĖdicas de Porto Alegre, MS em Ciĺncias BiolÛgicas:Bioquìmica, Doutoranda doProgramadePÛs-GraduaÁ,,oem Inform·ticanaEducaÁ,,odaUFRGS.

E-mail:anzevedo@fffcmpa.tche.br, http://www.fffcmpa.tche.br

Maria Suzana Marc Amoretti

Professora, doutoraemLing, İstica, SemiÛticaeCiĺncias Cognitivas(UniversitĖde Limoges/FranÁa), pesquisadoradoCNPqeprofessoradoCursodeDoutoradoem Inform·ticanaEducaÁ,,odaUFRGS.

E-mail:suzana@pgie.ufrgs.br http://www.planeta.terra.com.br/educacao/suzana

Maria Isabel Timm

Jornalista, Especialistaem ComunicaÁ,o(PPGCOM-UFRGS), Doutorandaem Inform·tica na EducaÁ,,o (UFRGS), Coordenadora Operacional do N'cleo de MultimldiaeEnsinoaDist,nciadaEscoladeEngenhariadaUFRGS,.

E-mail:betatimm@ufrgs.br http://www.nmead.ufrgs.br

Milton Antonio Zaro

Fl̀sico, PhDem MetalurgiaFl̀sica(UFSC), CoordenadorPedagÛgicodoN cleode MultimldiaeEnsinoaDist,nciadaEscoladeEngenhariadaUFRGS.

E-mail:zaro@ufrgs.br, http://www.nmead.ufrgs.br 